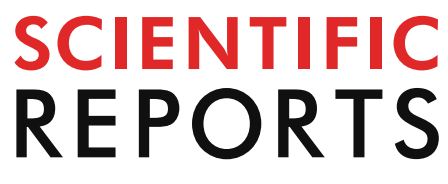

natureresearch

Check for updates

\title{
Beta-blockers
}

\section{and renin-angiotensin system inhibitors in acute myocardial infarction managed with inhospital coronary revascularization}

\author{
Hui Wen Sim ${ }^{1,2,14}$, Huili Zheng ${ }^{3,14}$, A. Mark Richards ${ }^{1,4,5}$, Ruth W. Chen ${ }^{6}$, \\ Anders Sahlen 7,8 , Khung-Keong Yeo ${ }^{7}$, Jack W. Tan ${ }^{7}$, Terrance Chua ${ }^{7}$, Huay Cheem Tan ${ }^{1,4}$, \\ Tiong Cheng Yeo ${ }^{1,4}$, Hee Hwa Ho ${ }^{6}$, Boon-Wah Liew ${ }^{9}$, Ling Li Foo ${ }^{3}$, Chi-Hang Lee ${ }^{1,4,5,15}$, \\ Derek J. Hausenloy ${ }^{4,7,9,10,11,12,13,15}$ \& Mark Y. Chan ${ }^{1,4,5,15 \bowtie}$
}

Pivotal trials of beta-blockers (BB) and angiotensin converting enzyme inhibitors/angiotensin receptor blockers (ACEI/ARB) in acute myocardial infarction (AMI) were largely conducted prior to the widespread adoption of early revascularization. A total of 15,073 patients with AMI who underwent inhospital coronary revascularization from January 2007 to December 2013 were analyzed. At 12 months, BB was significantly associated with a lower incidence of major adverse cardiovascular events (MACE, adjusted $\mathrm{HR} 0.80,95 \% \mathrm{Cl} 0.70-0.93$ ) and all-cause mortality (adjusted $\mathrm{HR} 0.69,95 \% \mathrm{Cl}$ 0.55-0.88), while ACEI/ARB was significantly associated with lower all-cause mortality (adjusted HR $0.80,95 \% \mathrm{Cl} 0.66-0.98$ ) and heart failure (HF) hospitalization (adjusted $\mathrm{HR} 0.80,95 \% \mathrm{Cl} 0.68-0.95$ ). Combined BB and ACEI/ARB use was associated with the lowest incidence of MACE (adjusted HR 0.70, $95 \% \mathrm{Cl} 0.57-0.86$ ), all-cause mortality (adjusted $\mathrm{HR} 0.55,95 \% \mathrm{Cl} 0.40-0.77$ ) and $\mathrm{HF}$ hospitalization (adjusted $\mathrm{HR} 0.64,95 \% \mathrm{Cl} 0.48-0.86$ ). This were consistent for left ventricular ejection fraction $<50 \%$ or $\geq 50 \%$. In conclusion, in AMI managed with revascularization, both BB and ACEI/ARB were associated with a lower incidence of 12-month all-cause mortality. Combined BB and ACEI/ARB was associated with the lowest incidence of all-cause mortality and HF hospitalization.

Beta-blockers (BB) and angiotensin-converting enzyme inhibitors/angiotensin receptor blockers (ACEI/ARB) have been the cornerstone of treatment for acute myocardial infarction (AMI) due to their salutary effects on short- and long-term mortality. However, the majority of clinical trials showing benefit with BB and ACEI/ $\mathrm{ARB}$ in $\mathrm{AMI}$ were performed before the widespread adoption of primary percutaneous coronary intervention

\footnotetext{
${ }^{1}$ Department of Cardiology, National University Heart Centre Singapore, 1E Kent Ridge Road, NUHS Tower Block, Level 9, Singapore 119228, Singapore. ${ }^{2}$ Department of Medicine, Ng Teng Fong General Hospital, 1 Jurong East Street 21, Singapore 609606, Singapore. ${ }^{3}$ Health Promotion Board, National Registry of Disease Office, 3 Second Hospital Ave, Singapore 168937, Singapore. ${ }^{4}$ Yong Loo Lin School of Medicine, National University of Singapore, 10 Medical Dr, Singapore 117597, Singapore. ${ }^{5}$ Cardiovascular Research Institute, 1E Kent Ridge Road, Singapore 119228, Singapore. ${ }^{6}$ Department of Cardiology, Tan Tock Seng Hospital, 11 Jln Tan Tock Seng, Singapore 308433, Singapore. ${ }^{7}$ National Heart Centre Singapore, 5 Hospital Dr, Singapore 169609, Singapore. ${ }^{8}$ Karolinska Institutet, Stockholm, Sweden. ${ }^{9}$ Department of Cardiology, Changi General Hospital, 2 Simei Street 3, Singapore 529889, Singapore. ${ }^{10}$ Cardiovascular and Metabolic Disorders Program, Duke-National University of Singapore Medical School, Singapore, Singapore. ${ }^{11}$ National Heart Research Institute Singapore, National Heart Centre, Singapore, Singapore. ${ }^{12}$ The Hatter Cardiovascular Institute, University College London, London, UK. ${ }^{13}$ Cardiovascular Research Center, College of Medical and Health Sciences, Asia University, Taichung City, Taiwan. ${ }^{14}$ These authors contributed equally: Hui Wen Sim and Huili Zheng. ${ }^{15}$ These authors jointly supervised this work: Chi-Hang Lee, Derek J. Hausenloy and MarkY.Chan. ${ }^{\circledR}$ email: mark_chan@nuhs.edu.sg
} 
(PCI) for ST-segment elevation MI (STEMI) and early invasive management for non-STEMI ${ }^{1-4}$. Even with more contemporary clinical trials of ACEI/ARB, many patients may not have undergone early revascularization ${ }^{5-7}$.

Contemporary observational data suggest an absence of mortality benefit from BB among patients with AMI and preserved left ventricular ejection fraction $(\mathrm{LVEF})^{8-12}$. A meta-analysis found that BB reduced mortality in the pre-reperfusion era but not in reperfusion $\mathrm{era}^{13}$. As a consequence, the European Society of Cardiology has downgraded its recommendation of routine BB administration for AMI from class I to class IIa ${ }^{14-16}$. In contrast, $\mathrm{ACEI} / \mathrm{ARB}$ have been shown to reduce mortality in AMI but a class I recommendation is only assigned to patients with anterior myocardial infarction (MI), heart failure (HF) and/or reduced $L_{V E F}{ }^{3-6,14-16}$. In patients with recent AMI, chronic upregulation of neurohormonal activity in the sympathetic nervous system and renin-angiotensin-aldosterone system exerts deleterious effect on the myocardium, leading to pump failure. BB and ACEI/ ARB downregulate sympathetic and renin-angiotensin system overactivity to improve left ventricular function and long-term clinical outcome $e^{17,18}$.

As such, there is a need to reassess the role of $\mathrm{BB}$ and ACEI/ARB in this contemporary treatment era of routine early revascularization for AMI. Therefore, we evaluated the association of BB and ACEI/ARB with 12-month mortality, HF hospitalization and MI in patients with AMI who had undergone inhospital coronary revascularization.

\section{Methods}

Data sources and study population. Patients diagnosed with STEMI or non-STEMI during their index admission from January 2007 to December 2013 were identified from the Singapore Myocardial Infarct Registry (SMIR, https://www.nrdo.gov.sg/publications/ami). AMI cases (International Classification of Diseases Ninth Revision and Tenth Revision) were identified from hospital discharge records, troponin test results, reimbursement claims and the national death registry by trained coordinators from the SMIR. Healthcare legislature in Singapore mandates that all patients diagnosed with AMI are enrolled in the SMIR with the exception of patients who opt out of enrolment. This study complies to the Helsinki declaration and was approved by the National Healthcare Group Domain Specific Review Board which allowed for a waiver of written informed consent on condition that all analyses were performed onsite at the SMIR using de-identified data.

We included all patients with a primary diagnosis of AMI and who received inhospital coronary revascularization by PCI or coronary artery bypass graft surgery (CABG) during the index hospitalization. We excluded (1) patients who were admitted for non-AMI condition but had AMI during hospitalization, (2) AMI that were not clearly classified (not STEMI or non-STEMI), (3) patients who did not receive inhospital revascularization, and (4) patients who died during index hospitalization.

Data collection and clinical outcomes. Information on demographics, co-morbidities, history of coronary revascularization, clinical presentation, inpatient laboratory values, LVEF and pharmacotherapy on discharge were prospectively collected by trained coordinators according to a standardized case report form (https://www.nrdo.gov.sg/docs/default-source/Disease-Notification-AMI/nrdo-f004-09b-(smir-notificationform)web.pdf?sfvrsn=0). Prior to 2008, LVEF data in the registry was captured in binary format (LVEF $<50 \%$ vs $\geq 50 \%$ ). From 2008 onwards, LVEF was captured as continuous data. The outcome of interest was major adverse cardiovascular events (MACE), which we defined as a composite of all-cause mortality, hospitalization for HF or hospitalization for MI, and the individual component endpoints. Death endpoints were ascertained through data linkage with the Ministry of Home Affairs Death Registry while MI hospitalization and HF hospitalization were ascertained by linking SMIR data with the Ministry of Health Mediclaims data. Only the first hospitalization for HF or MI after discharge was included and time to hospitalization was computed as the number of days from the discharge date of the index admission to the readmission date.

Statistical analysis. For descriptive analyses, we compared baseline demographic and clinical characteristics of patients stratified to $\mathrm{BB}$ versus no $\mathrm{BB}$ and $\mathrm{ACEI} / \mathrm{ARB}$ versus no ACEI/ARB. Categorical variables are shown using frequencies and percentages, and continuous variables are presented using median and interquartile range. Differences between the groups were compared by using Chi-square test for categorical variables and Mann-Whitney-Wilcoxon nonparametric test for continuous variables. Multivariable Cox proportional hazard regression models were constructed to estimate the hazard ratio (HR) and 95\% confidence interval (CI) for the risk of composite endpoint, all-cause mortality, MI and HF hospitalization, for patients who were given (1) BB and those who were not given (reference group) and (2) ACEI/ARB compared to those who were not given these medications (reference group). Included in the multivariable models were age, gender, ethnicity, hypertension, diabetes, hyperlipidemia, history of MI/PCI/CABG, smoking status, Killip class on admission, creatinine level on admission and in-hospital $\mathrm{LVEF}<50 \%$. We further constructed another similar multivariable Cox proportional hazard regression model for patients who received both $\mathrm{BB}$ and ACEI/ARB (BB + ACEI/ARB), BB only, ACEI/ARB only, comparing them with the reference group of patients were on neither BB nor ACEI/ARB (no BB + no ACEI/ARB group). Competing risks from death was accounted for all hospitalization outcomes ${ }^{19}$. Secondary subgroup analysis examined clinical outcomes stratified by the following categories: types of AMI (STEMI or NSTEMI), age (<65 years old or $\geq 65$ years old), sex (male or female), history of diabetes, history of hypertension, Killip class on presentation (I/II or III/III), LVEF during hospitalization (<50\% or $\geq 50 \%)$, PCI during hospitalization and CABG during hospitalization. All tests were performed with STATA SE software, version 13 . For all analyses, a two-sided $P<0.05$ was considered statistically significant. 


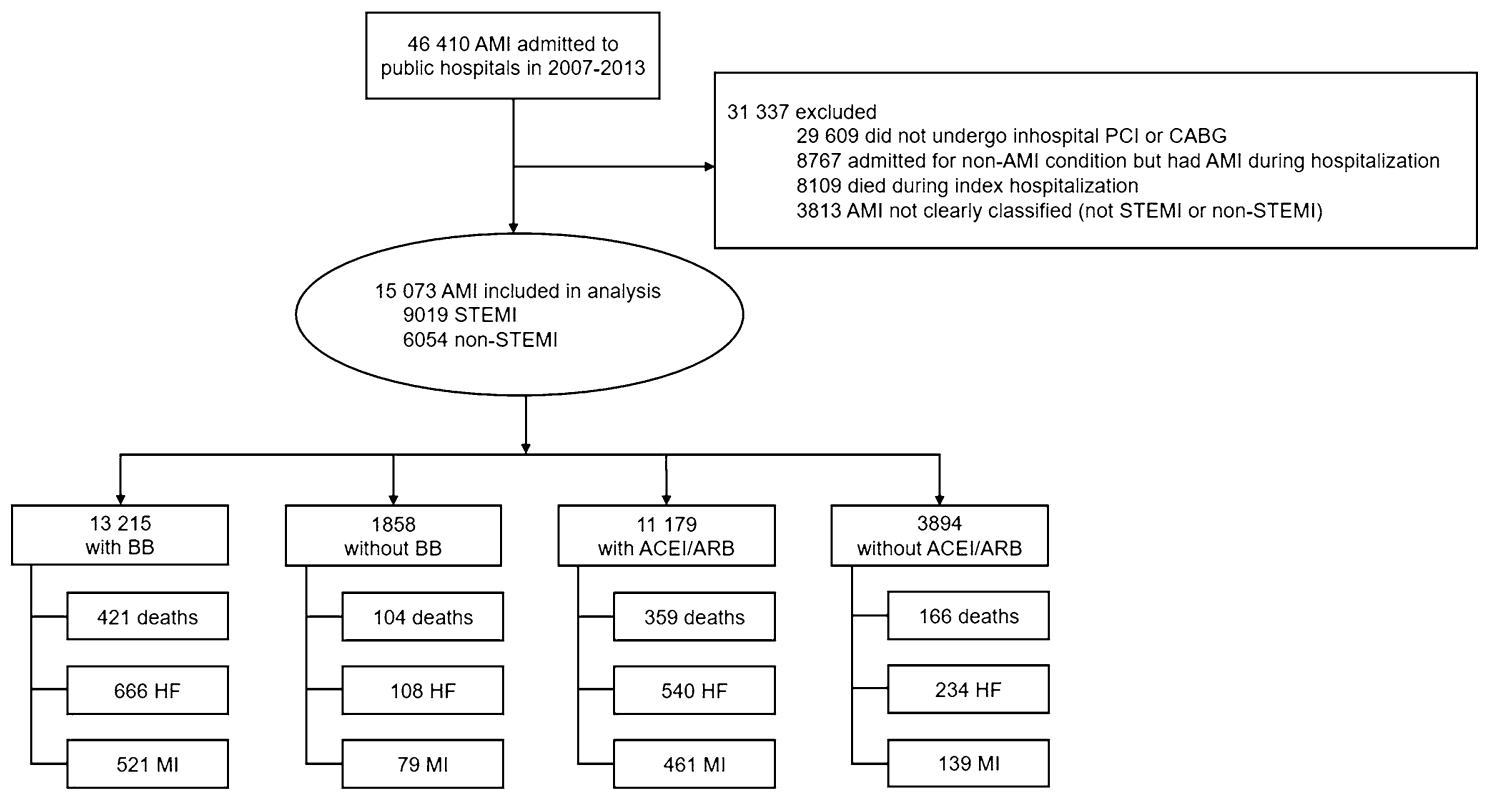

Figure 1. Study flow diagram. $A C E I / A R B$ Angiotensin converting enzyme inhibitors/angiotensin receptor blockers, $A M I$ acute myocardial infarction, $B B$ beta-blockers, $C A B G$ coronary artery bypass graft, $C I$ confidence interval, $H F$ heart failure, $H R$ hazard ratio, $L V E F$ left ventricular ejection fraction, $M A C E$ major adverse cardiovascular events, $M I$ myocardial infarction, NSTEMI non-ST-segment elevation myocardial infarction, STEMI ST-segment elevation myocardial infarction, PCI percutaneous coronary intervention.

\section{Results}

Baseline characteristics. Out of 46,410 patients with AMI from January 2007 to December 2013, 15,073 patients were included in the present analysis, of which 9,019 (59.8\%) patients had STEMI and 6,054 (40.2\%) of patients had non-STEMI (Fig. 1). All patients in this study cohort underwent inpatient revascularization: 14,168 (94\%) had PCI while 1,024 (6.8\%) had CABG. Among these patients, $119(0.8 \%)$ had both PCI and CABG during their index hospitalization. The median time from admission to revascularization was 1 day (interquartile range $0-2$ days). A total of 13,215 (87.7\%) patients were on BB and 11,179 (74.2\%) patients were on ACEI/ARB. Among them, 10,063 (66.8\%) were on both BB and ACEI/ARB (BB + ACEI/ARB), 3,152 (20.9\%) were only on $\mathrm{BB}$ (BB only), 1,116 (7.4\%) were only on ACEI/ARB (ACEI/ARB only), and 742 (4.9\%) were on neither BB nor $\mathrm{ACEI} / \mathrm{ARB}$ (no BB + no ACEI/ARB group).

Table 1 illustrates the baseline characteristics of the study cohort. Those who were prescribed BB were younger, more likely to be men, have hypertension and more likely to have impaired LVEF $<50 \%$ than those who were not. Patients who were prescribed ACEI/ARB were more likely to have diabetes, hypertension, hyperlipidemia, previous MI, previous PCI, previous CABG and STEMI at presentation than those who were not.

At 12 months of follow up, MACE occurred in 1,671 (11.1\%) patients. A total of 525 (3.5\%) patients died, 774 (5.1\%) patients were hospitalized for HF and 600 (4.0\%) patients were hospitalized for MI. BB use at discharge was associated with a lower incidence of the combined MACE endpoint (adjusted HR 0.80, 95\% CI 0.70-0.93) and all-cause mortality (adjusted HR $0.69,95 \%$ CI 0.55-0.88) while ACEI/ARB use at discharge was associated with lower all-cause mortality (adjusted HR 0.80, 95\% CI 0.66-0.98) and lower hospitalization for HF (adjusted HR $0.80,95 \%$ CI $0.68-0.95$ ). Neither BB nor ACEI/ARB at discharge was associated with lower incidence of hospitalization for MI (Table 2). We next compared MACE and the individual endpoints among the following four groups: $\mathrm{BB}+\mathrm{ACEI} / \mathrm{ARB}, \mathrm{BB}$ only, $\mathrm{ACEI} / \mathrm{ARB}$ only and no $\mathrm{BB}+$ no $\mathrm{ACEI} / \mathrm{ARB}$ group (reference group). The BB + ACEI/ARB group had the lowest incidence of MACE (adjusted HR 0.70, 95\% CI 0.57-0.86), all-cause mortality (adjusted HR 0.55, 95\% CI 0.40-0.77) and hospitalization for HF (adjusted HR 0.64, 95\% CI 0.48-0.86) among all four groups. BB only was associated with a lower incidence of MACE (adjusted HR 0.74, 95\% CI $0.59-0.92$ ) and all-cause mortality (adjusted HR 0.64, 95\% CI 0.45-0.92) than the no ACEI/ARB + no BB group. ACEI/ARB-only was associated with a lower incidence of hospitalization for HF (adjusted HR 0.67, 95\% CI 0.45 -0 0.99) than the no ACEI/ARB + no BB group. There was no difference in the incidence of MI in either of the first three treatment groups when compared with the no BB + no ACE/ABR group (Table 2).

The treatment effect for the composite MACE and individual endpoints of all-cause mortality, MI and HF hospitalization was largely consistent and homogenous across all subgroups for BB (Fig. 2) and ACEI/ARB (Fig. 3). Of note, the association of BB at discharge with lower mortality (Fig. 2b) and ACEI/ARB at discharge lower hospitalization for HF (Fig. 3c) was consistent across LVEF subgroups of $<50 \%$ and $\geq 50 \%$. The subgroup analysis showed that the risk of mortality and MI hospitalization associated with BB prescribed at discharge were higher in the subgroup who underwent revascularization with CABG as compared to PCI. Conversely, HF hospitalization associated with $\mathrm{BB}$ was lower in patients who underwent CABG as compared to PCI. In patients who receive ACEI/ARB, the risk of mortality, HF and MI hospitalization were consistently higher in the subgroup who underwent revascularization with CABG as compared to PCI. 


\begin{tabular}{|c|c|c|c|c|c|c|c|}
\hline & \multirow{2}{*}{$\begin{array}{l}\text { All patients } \\
(\mathrm{N}=15,073)\end{array}$} & \multicolumn{3}{|l|}{ Discharge BB } & \multicolumn{3}{|c|}{ Discharge ACEI/ARB } \\
\hline & & Yes $(n=13,215)$ & No $(n=1,858)$ & $P$ value & Yes $(n=11,179)$ & No $(n=3,894)$ & $P$ value \\
\hline \multicolumn{8}{|l|}{ Baseline demographics } \\
\hline $\begin{array}{l}\text { Age in years, median } \\
\text { (IQR) }\end{array}$ & $58(51-66)$ & $58(51-66)$ & $60(52-70)$ & $<.001$ & $58(51-66)$ & $58(51-66)$ & .601 \\
\hline Male, n (\%) & $12,537(83.2)$ & $11,036(83.5)$ & $1,501(80.8)$ & .003 & $9,320(83.4)$ & $3,217(82.6)$ & 0.28 \\
\hline \multicolumn{8}{|l|}{ Race } \\
\hline Chinese, $\mathrm{n}(\%)$ & $9,422(62.5)$ & $8,254(62.5)$ & $1,168(62.9)$ & \multirow{4}{*}{.908} & $6,955(62.2)$ & $2,467(63.4)$ & \multirow{4}{*}{.020} \\
\hline Malay, n (\%) & $2,901(19.3)$ & $2,549(19.3)$ & $352(19.0)$ & & $2,130(19.1)$ & $771(19.8)$ & \\
\hline Indian, $\mathrm{n}(\%)$ & $2,499(16.6)$ & $2,195(16.6)$ & $304(16.4)$ & & $1,914(17.1)$ & $585(15.0)$ & \\
\hline Others & $251(1.6)$ & $217(1.6)$ & $34(1.8)$ & & $180(1.6)$ & $71(1.8)$ & \\
\hline $\begin{array}{l}\text { Current/former } \\
\text { smoker, n (\%) }\end{array}$ & $9,274(61.7)$ & $8,048(61.1)$ & $1,226(66.2)$ & $<.001$ & 6,869 (61.6) & $2,405(62.1)$ & 0.60 \\
\hline $\begin{array}{l}\text { History of diabetes, } \\
\mathrm{n}(\%)\end{array}$ & $4,725(31.4)$ & $4,162(31.5)$ & $563(30.4)$ & .31 & $3,605(32.3)$ & $1,120(28.8)$ & $<.001$ \\
\hline $\begin{array}{l}\text { History of hyperten- } \\
\text { sion, } n(\%)\end{array}$ & $8,661(57.5)$ & $7,683(58.2)$ & $978(52.8)$ & $<.001$ & $6,771(60.6)$ & $1,890(48.6)$ & $<.001$ \\
\hline $\begin{array}{l}\text { Hyperlipidemia, } \\
\text { n (\%) }\end{array}$ & $7,884(52.4)$ & $6,894(52.2)$ & $990(53.4)$ & .35 & $5,930(53.1)$ & $1,954(50.2)$ & .002 \\
\hline History of MI, n (\%) & 2,351 (15.6) & $2,036(15.4)$ & $315(17.0)$ & .09 & $1,810(16.2)$ & $541(13.9)$ & .001 \\
\hline History of PCI, n (\%) & $2,126(14.2)$ & $1,860(14.1)$ & $266(14.4)$ & .76 & $1,655(14.9)$ & $471(12.1)$ & $<.001$ \\
\hline $\begin{array}{l}\text { History of CABG, } \\
\mathrm{n}(\%)\end{array}$ & $531(3.5)$ & $464(3.5)$ & $67(3.6)$ & .83 & $425(3.8)$ & $106(2.7)$ & .002 \\
\hline \multicolumn{8}{|l|}{ Clinical presentation } \\
\hline STEMI & $9,019(59.8)$ & $7,950(60.2)$ & $1,069(57.5)$ & \multirow{2}{*}{.03} & $6,874(61.5)$ & $2,145(55.1)$ & \multirow{2}{*}{$<.001$} \\
\hline Non-STEMI & $6,054(40.2)$ & $5,265(39.8)$ & $789(42.5)$ & & $4,305(38.5)$ & $1,749(44.9)$ & \\
\hline PCI, n (\%) & $14,168(94.0)$ & $12,488(94.5)$ & $1,680(90.4)$ & $<.001$ & $10,870(97.2)$ & $3,298(84.7)$ & $<.001$ \\
\hline CABG, n (\%) & $1,024(6.8)$ & $821(6.2)$ & $203(10.9)$ & $<.001$ & $355(3.2)$ & $669(17.2)$ & $<.001$ \\
\hline $\begin{array}{l}\text { Primary PCI among } \\
\text { STEMI, } \mathrm{n}(\%)\end{array}$ & $7,577(84.0)$ & 6,688 (84.1) & $889(83.2)$ & .42 & $5,801(84.4)$ & $1,776(82.8)$ & .08 \\
\hline Killip class I/II, n (\%) & $13,992(92.8)$ & $12,289(93.0)$ & $1,703(91.7)$ & \multirow[b]{2}{*}{.04} & $10,449(93.5)$ & $3,543(91.0)$ & \multirow[b]{2}{*}{$<.001$} \\
\hline $\begin{array}{l}\text { Killip class III/IV, } \\
\mathrm{n}(\%)\end{array}$ & $1,079(7.2)$ & $924(7.0)$ & $155(8.3)$ & & $729(6.5)$ & $350(9.0)$ & \\
\hline $\begin{array}{l}\text { Serum creatinine } \\
\text { in umol/L, median } \\
\text { (IQR) }\end{array}$ & $88(75-105)$ & $88(75-105)$ & $90(76-108)$ & $<.001$ & $87(74-104)$ & $89(76-109)$ & $<.001$ \\
\hline LVEF < 50\%, n (\%) & $7,321(54.2)$ & $6,468(54.6)$ & $853(51.0)$ & .005 & $5,413(53.8)$ & $1,908(55.3)$ & .14 \\
\hline $\operatorname{LVEF}<30 \%, \mathrm{n}(\%)^{\mathrm{a}}$ & $1,045(8.5)$ & $902(8.3)$ & $143(9.5)$ & .118 & $723(7.9)$ & $322(10.3)$ & $<.001$ \\
\hline $\begin{array}{l}\text { LVEF in \%, median } \\
(\mathrm{IQR})^{\mathrm{a}}\end{array}$ & $45(38-55)$ & $50(38-55)$ & $45(38-55)$ & .07 & $45(35-55)$ & $45(40-55)$ & .005 \\
\hline \multicolumn{8}{|c|}{ Medication given at discharge } \\
\hline Aspirin, $\mathrm{n}(\%)$ & $14,509(96.3)$ & $12,767(96.6)$ & $1,742(93.8)$ & $<.001$ & $10,860(97.2)$ & 3,649 (93.7) & $<.001$ \\
\hline $\begin{array}{l}\text { P2Y12 inhibitors, } \\
\text { n (\%) }\end{array}$ & $14,433(95.8)$ & $12,685(96.0)$ & $1,748(94.2)$ & $<.001$ & $10,943(97.9)$ & $3,490(89.7)$ & $<.001$ \\
\hline Beta-blocker, n (\%) & $13,215(87.7)$ & - & - & - & $10,063(90.0)$ & $3,152(81.0)$ & $<.001$ \\
\hline ACEI/ARB, n (\%) & $11,179(74.2)$ & $10,063(76.2)$ & $1,116(60.1)$ & $<.001$ & - & - & - \\
\hline $\begin{array}{l}\text { Lipid lowering } \\
\text { drugs, } n(\%)\end{array}$ & $14,725(97.7)$ & $12,952(98.0)$ & $1,773(95.4)$ & $<.001$ & $10,982(98.2)$ & $3,743(96.1)$ & $<.001$ \\
\hline
\end{tabular}

Table 1. Baseline demographics, clinical presentation and medication given at discharge. $A C E I$ angiotensin converting enzyme inhibitor, $A R B$ angiotensin receptor blocker, $C A B G$ coronary artery bypass graft, $H F$ heart failure, $I Q R$ interquartile range, $L V E F$ left ventricular ejection fraction, $M I$ myocardial infarction, $P C I$ percutaneous coronary intervention, STEMI ST-segment elevation myocardial infarction. ${ }^{a}$ Data was from 2008 to 2013 .

\section{Discussion}

$\mathrm{BB}$ and $\mathrm{ACEI} / \mathrm{ARB}$ may confer additive effects on survival in patients with AMI via different mechanisms: BB inhibits sympathetic activity and guards against arrhythmic deaths while ACEI/ARB modifies cardiac remodelling and reduces HF mortality ${ }^{20}$. In this contemporary cohort of patients with AMI undergoing inhospital revascularization, we observed that prescription of either BB or ACEI/ARB at discharge was associated with a lower incidence of 12-month all-cause mortality compared with patients who received neither drug at discharge, even after adjusting for relevant confounding variables. ACEI/ARB use at discharge, with or without BB, was associated with a reduced incidence of hospitalization for HF. Neither BB nor ACEI/ARB was associated with incidence of 


\begin{tabular}{|c|c|c|c|c|c|c|c|c|}
\hline \multirow[b]{2}{*}{ Adverse events } & \multicolumn{2}{|l|}{ BB } & \multicolumn{2}{|l|}{ ACEI/ARB } & \multicolumn{4}{|c|}{$\mathrm{BB}$ and ACEI/ARB } \\
\hline & Yes $(n=13,215)$ & No $(n=1,858)$ & Yes $(n=11,179)$ & No $(n=3,894)$ & $\begin{array}{l}\text { BB + ACEI/ARB } \\
(n=10,063)\end{array}$ & BB only $(n=3,152)$ & $\begin{array}{l}\text { ACEI/ARB only } \\
(n=1,116)\end{array}$ & $\begin{array}{l}\text { No BB + No ACEI/ } \\
\text { ARB }(n=742)\end{array}$ \\
\hline \multicolumn{9}{|l|}{ MACE } \\
\hline $\begin{array}{l}\text { Crude event rates, } \\
\mathrm{n}(\%)\end{array}$ & $1,418(10.7)$ & $253(13.6)$ & $1,206(10.8)$ & $465(11.9)$ & $1,065(10.6)$ & $353(11.2)$ & $141(12.6)$ & $112(15.1)$ \\
\hline $\begin{array}{l}\text { Unadjusted HR } \\
(95 \% \mathrm{CI})\end{array}$ & $0.77(0.68-0.89)$ & $1.00(\mathrm{ref})$ & $0.89(0.80-0.99)$ & 1.00 (ref) & $0.68(0.56-0.82)$ & $0.72(0.59-0.90)$ & $0.82(0.64-1.05)$ & $1.00($ ref $)$ \\
\hline $\begin{array}{l}\text { Adjusted HR (95\% } \\
\text { CI) }{ }^{\mathrm{a}}\end{array}$ & $0.80(0.70-0.93)$ & $1.00(\mathrm{ref})$ & $0.91(0.81-1.02)$ & $1.00(\mathrm{ref})$ & $0.70(0.57-0.86)$ & $0.74(0.59-0.92)$ & $0.80(0.62-1.05)$ & $1.00(\mathrm{ref})$ \\
\hline \multicolumn{9}{|l|}{ All-cause mortality } \\
\hline $\begin{array}{l}\text { Crude event rates, } \\
\mathrm{n}(\%)\end{array}$ & $421(3.2)$ & $104(5.6)$ & $359(3.2)$ & $166(4.3)$ & $305(3.0)$ & $116(3.7)$ & $54(4.8)$ & $50(6.7)$ \\
\hline $\begin{array}{l}\text { Unadjusted HR } \\
(95 \% \mathrm{CI})\end{array}$ & $0.57(0.46-0.71)$ & $1.00(\mathrm{ref})$ & $0.75(0.62-0.90)$ & $1.00(\mathrm{ref})$ & $0.44(0.33-0.60)$ & $0.54(0.39-0.76)$ & $0.71(0.48-1.04)$ & $1.00(\mathrm{ref})$ \\
\hline $\begin{array}{l}\text { Adjusted HR (95\% } \\
\text { CI) }\end{array}$ & $0.69(0.55-0.88)$ & $1.00(\mathrm{ref})$ & $0.80(0.66-0.98)$ & $1.00(\mathrm{ref})$ & $0.55(0.40-0.77)$ & $0.64(0.45-0.92)$ & $0.72(0.47-1.10)$ & $1.00(\mathrm{ref})$ \\
\hline \multicolumn{9}{|l|}{ HF hospitalization } \\
\hline $\begin{array}{l}\text { Crude event rates, } \\
\mathrm{n}(\%)\end{array}$ & $666(5.0)$ & $108(5.8)$ & $540(4.8)$ & $234(6.0)$ & $488(4.9)$ & $178(5.7)$ & $52(4.7)$ & $56(7.6)$ \\
\hline $\begin{array}{l}\text { Unadjusted HR } \\
(95 \% \mathrm{CI})\end{array}$ & $0.86(0.70-1.05)$ & $1.00(\mathrm{ref})$ & $0.80(0.68-0.93)$ & $1.00(\mathrm{ref})$ & $0.63(0.48-0.83)$ & $0.74(0.55-1.00)$ & $0.61(0.42-0.89)$ & $1.00(\mathrm{ref})$ \\
\hline $\begin{array}{l}\text { Adjusted HR (95\% } \\
\text { CI) })^{\mathrm{a}}\end{array}$ & $0.84(0.68-1.03)$ & $1.00(\mathrm{ref})$ & $0.80(0.68-0.95)$ & $1.00(\mathrm{ref})$ & $0.64(0.48-0.86)$ & $0.76(0.55-1.04)$ & $0.67(0.45-0.99)$ & $1.00(\mathrm{ref})$ \\
\hline \multicolumn{9}{|l|}{ MI hospitalization } \\
\hline $\begin{array}{l}\text { Crude event rates, } \\
\text { n (\%) }\end{array}$ & $521(3.9)$ & $79(4.3)$ & $461(4.1)$ & $139(3.6)$ & $403(4.0)$ & $118(3.7)$ & $58(5.2)$ & $21(2.8)$ \\
\hline $\begin{array}{l}\text { Unadjusted HR } \\
(95 \% \mathrm{CI})\end{array}$ & $0.92(0.73-1.17)$ & $1.00(\mathrm{ref})$ & $1.16(0.96-1.40)$ & $1.00(\mathrm{ref})$ & $1.42(0.91-2.20)$ & $1.32(0.83-2.11)$ & $1.85(1.12-3.06)$ & $1.00(\mathrm{ref})$ \\
\hline $\begin{array}{l}\text { Adjusted HR (95\% } \\
\text { CI) }\end{array}$ & $0.98(0.76-1.28)$ & $1.00(\mathrm{ref})$ & $1.20(0.97-1.49)$ & $1.00(\mathrm{ref})$ & $1.44(0.89-2.33)$ & $1.27(0.77-2.12)$ & $1.71(0.99-2.96)$ & $1.00(\mathrm{ref})$ \\
\hline
\end{tabular}

Table 2. Crude and adjusted event rates among patients given $\mathrm{BB}$ and $\mathrm{ACEI} / \mathrm{ARB}$ at discharge. ACEI angiotensin converting enzyme inhibitor, $A R B$ angiotensin receptor blocker, $C I$ confidence interval, $H F$ heart failure, $H R$ hazard ratio, $M I$ myocardial infarction. a Adjusted for age, sex, race, history of diabetes, history of hypertension, history of hyperlipidemia, history of myocardial infarction/percutaneous coronary intervention/ coronary artery bypass graft, smoking status, Killip class on presentation, serum creatinine on presentation, $\mathrm{LVEF}<50 \%$ during hospitalization.

MI. The prescription of both BB and ACEI/ARB was associated with the lowest incidence of 12-month MACE compared with the prescription of $\mathrm{BB}$ only, $\mathrm{ACEI} / \mathrm{ARB}$ only or neither $\mathrm{BB}$ nor ACEI/ARB at discharge. These findings were consistent across subgroups of reduced $(<50 \%)$ and preserved $(\geq 50 \%)$ LVEF.

Pivotal trials of $\mathrm{BB}$ in $\mathrm{AMI}$ were largely conducted prior to routine invasive management of $\mathrm{AMI}^{1}$. The more contemporary CAPRICORN trial (Effect of Carvedilol on Outcome after Myocardial Infarction in Patients with Left Ventricular Dysfunction) demonstrated 23\% reduction in mortality for post-MI patients with reduced $\mathrm{LVEF}^{21}$. In addition, several contemporary AMI registries reported lower mortality in those treated with BB, but only in those with reduced LVEF or evidence of $\mathrm{HF}^{22,23}$. Conversely, for preserved LVEF, data from the Myocardial Ischaemia National Audit Project, Reduction of Atherothrombosis for Continued Health and French Registry on Acute ST-segment elevation, Non-ST elevation myocardial infarction registries and post hoc analysis from the CHARISMA trial demonstrated no mortality benefit associated with the routine use of $\mathrm{BB}^{8,9,11,24}$. Compared to our study cohort, these studies had lower use of secondary prevention drugs among their subjects and included patients who did not undergo revascularization. Ongoing randomized controlled trials of routine BB use in patients with AMI and preserved LVEF, including the REDUCE-SWEDEHEART trial (NCT03278509) and the REBOOT-CNIC trial (NTC03596385), will hopefully address this knowledge gap.

Historically, randomized controlled trials of ACEI/ARB have demonstrated a reduction in mortality and hospitalization for HF among patients with AMI and both reduced LVEF as well as preserved $\mathrm{LVEF}^{7,25}$. In contrast, several contemporary observational studies have found no reduction in mortality or hospitalization for HF associated with ACEI/ARB among patients with AMI and preserved LVEF ${ }^{26,27}$. Several unique features of our study population should be noted when compared with these aforementioned observational studies: there was a high prevalence of cardiovascular risk factors (31.4\% diabetes, $57.5 \%$ hypertension, and $52.4 \%$ hyperlipidemia), all study participants underwent inhospital revascularization, and we were able to compare clinical outcomes between patients prescribed combination treatment with BB + ACEI/ARB, BB only, ACEI/ARB only versus no $\mathrm{BB}+$ no ACEI/ARB. One other observational study that looked at AMI patients who underwent PCI in South Korea $(\mathrm{N}=33,390)$ found no significant differences in the adjusted risk of all-cause mortality for patients treated with $\mathrm{BB}+\mathrm{ACEI} / \mathrm{ARB}, \mathrm{BB}$-only, ACEI/ARB-only as compared to non-treatment ${ }^{28}$. However, they only included 
a. MACE for $B B$

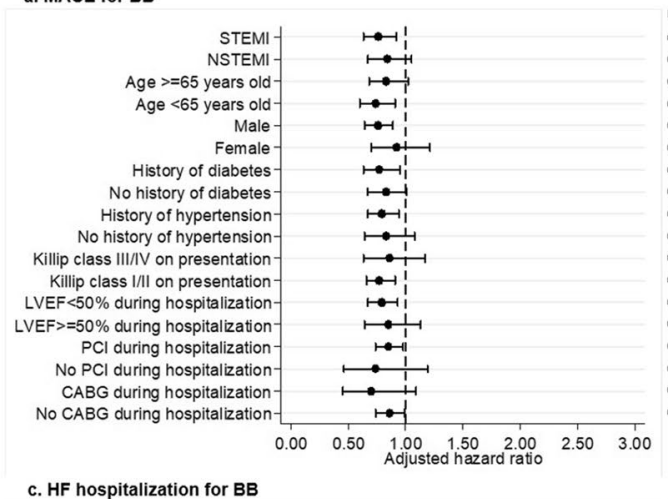

c. HF hospitalization for BB

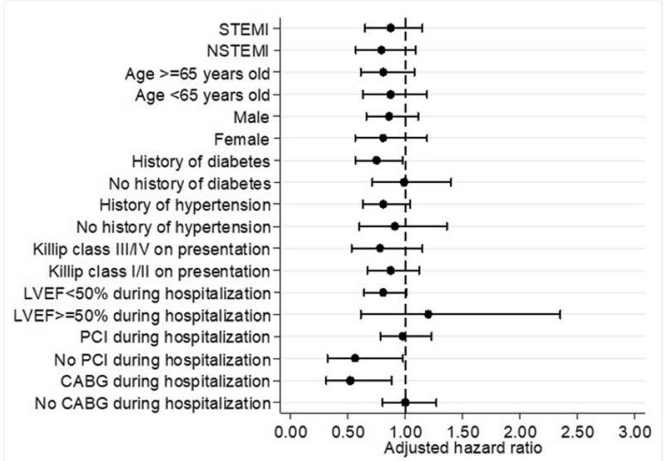

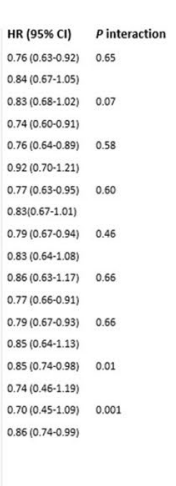

HR (95\% Cl) $\quad P$ interaction

$0.87(0.65 \cdot 1.15) \quad 0.45$

0.79 (0.57-1.09)

$0.81(0.61-1.08) \quad 0.93$

$0.87(0.63-1.19)$

$0.86(0.66-1.11) \quad 0.60$

$0.81(0.56-1.19)$

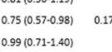

$0.81(0.65 \cdot 1.04) \quad 0.85$

$0.91(0.60-1.36)$

$0.78(0.53-1.1 .15) \quad 0.50$

$0.87(0.67-1.12)$

$0.81(0.64-1.01) \quad 0.6$

$1.20(0.61-2.35)$

$0.98(0.78 \cdot 1.23) \quad 0.24$

$0.56(0.32-0.98)$

$0.52(0.31-0.88) \quad 0.1$

$0.52(0.31-0.88)$ b. All-cause mortality for BB

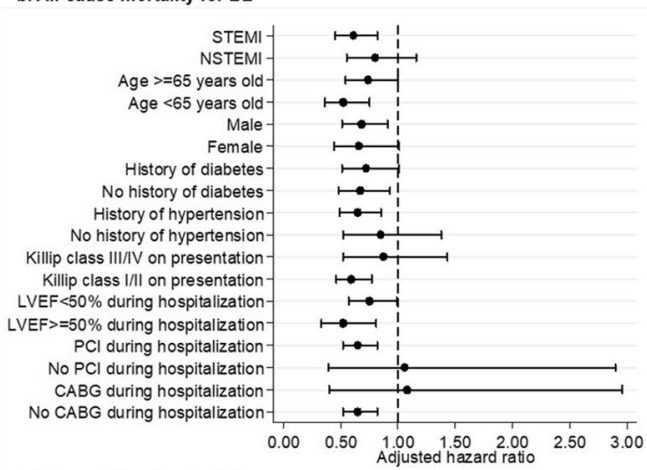

d. MI hospitalization for BB

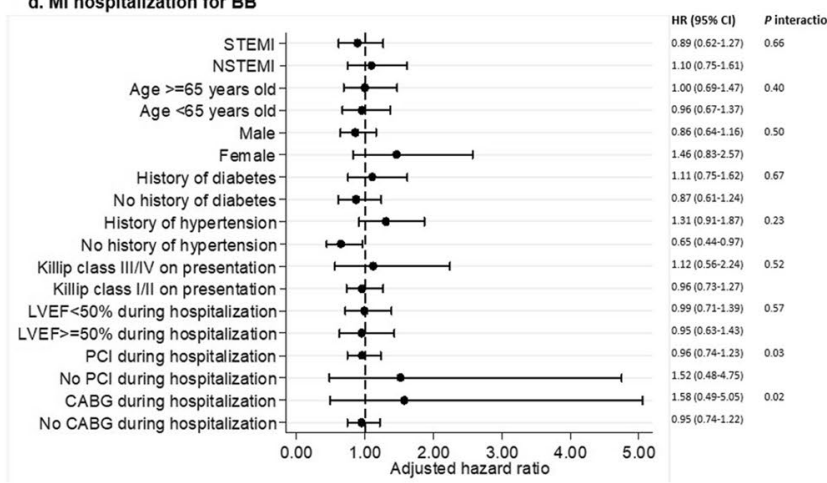

HR (95\% C1) $\rho$ interaction

$0.61(0.45-0.82) \quad 0.03$

$0.80(0.55-1.16)$

$0.74(0.54-1.00) \quad 0.11$

0.68 (0.51-0.91) 0.35

$0.66(0.44-1.01)$

$0.72(0.51-1.11) \quad 0.91$

$0.67(0.48-0.93)$

$65(0.49-0.85) \quad 0.23$

$0.85(0.52 \cdot 1.38) \quad 0.52-1.43) \quad 0.2$

$0.59(0.450 .077)$

$0.75(0.57-0.99) \quad 0.95$

$0.52(0.33-0.81)$

$0.65(0.52-0.82) \quad 0.25$

$1.06(0.39-2.90)$

$1.08(0.40-2.296) \quad 0.16$

$0.65(0.52-0.82)$

Figure 2. Adjusted risk of MACE among patients given $\mathrm{BB}$ at discharge compared to those not given $\mathrm{BB}$ at discharge (reference group) by subgroups. $A C E I / A R B$ Angiotensin converting enzyme inhibitors/angiotensin receptor blockers, $A M I$ acute myocardial infarction, $B B$ beta-blockers, $C A B G$ coronary artery bypass graft, $C I$ confidence interval, $H F$ heart failure, $H R$ hazard ratio, $L V E F$ left ventricular ejection fraction, MACE major adverse cardiovascular events, $M I$ myocardial infarction, NSTEMI non-ST-segment elevation myocardial infarction, STEMI ST-segment elevation myocardial infarction, PCI percutaneous coronary intervention. This figure was performed with STATA SE software, version 13.

patients who survived 30 days after the index procedure, which may result in low risk patients being selected. This study also did not examine other clinical outcomes such as hospitalization for HF or MI.

Several other findings in our study desire elaboration. First, we found that neither BB nor ACEI/ARB prescription at discharge was associated with a reduction in subsequent hospitalization for MI (Table 2). We postulate that only including revascularized AMI patients with high usage of dual antiplatelet therapy and lipid lowering drugs could have mitigated their risk of future MI, resulting in similar incidence of recurrent MI between patients who were and were not treated with $\mathrm{BB}$ or ACEI/ARB. Second, the subgroup analysis showed that the mortality benefit associated with $\mathrm{BB}$ (Fig. 2b) or ACEI/ARB (Fig. 3b) prescription at discharge was greater among patients in Killip class I/II at presentation compared with patients in Killip class III/IV at presentation (Interaction $p 0.02$ for $\mathrm{BB}$ and 0.005 for $\mathrm{ACEI} / \mathrm{ARB})$. Here, we hypothesize that a cautious approach is needed when prescribing either $\mathrm{BB}$ or $\mathrm{ACEI} / \mathrm{ARB}$ to patients with severe acute heart failure or cardiogenic shock. It is unknown whether patients in the higher Killip classes from our study might have been more susceptible to systemic arterial hypotension from early BB or ACEI/ARB use, worse HF from BB use or acute kidney injury and/or hyperkalemia from $\mathrm{ACEI} / \mathrm{ARB}$ use. We therefore recommend that early post-discharge monitoring of blood pressure, renal function and potassium be routine in this high risk group of patients. Third, BB and ACEI/ARB therapies were associated with better clinical outcomes despite the differences in baseline demographics between the group who were treated versus those who were not. Patients treated with BB had a lower risk profile (younger patients, fewer smokers) than those who were not treated with BB, whereas patients treated with ACEI/ARB had a higher risk profile (more diabetes, hypertension, hyperlipidemia, previous MI, previous PCI, and previous CABG) than those who were not treated with ACEI/ARB. These differences in baseline risk between treatment groups may have enhanced the benefit associated with BB use and diminished the benefit associated with ACEI/ARB use because of confounding. Forth, the subgroup analysis showed that in patients prescribed with BB or ACEI/ARB at discharge, risk of adverse clinical outcomes (except HF hospitalization in BB) was higher in the group who underwent revascularization with CABG as compared to PCI. It is likely that patients who underwent CABG were sicker at presentation due to multivessel coronary artery disease. They would also tend to have delayed intervention as compared to PCI resulting in loss of myocardium.

Our study represents a contemporary national registry with high standards of background care in pharmacotherapy. In addition, 24-h nationwide primary PCI has been the standard of care for STEMI in Singapore since 2007. To our knowledge, our cohort is the largest registry of AMI who underwent inhospital revascularization that study the association of $\mathrm{BB}$ and $\mathrm{ACEI} / \mathrm{ARB}$ with MACE, allowing robust subgroup comparisons. Our study 
a. MACE for ACEI/ARB

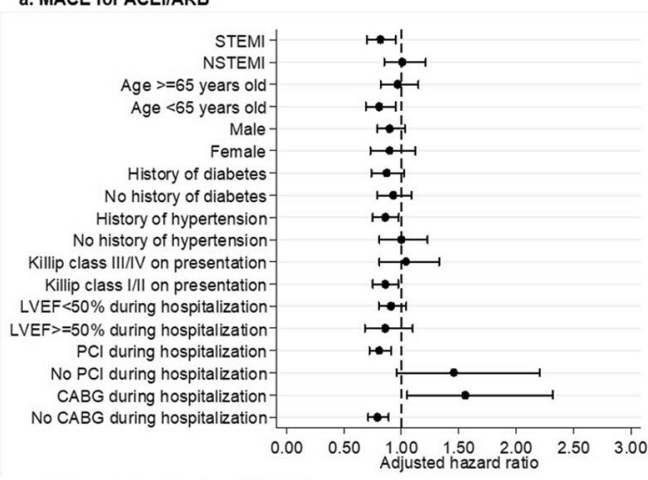

c. HF hospitalization for ACEI/ARB

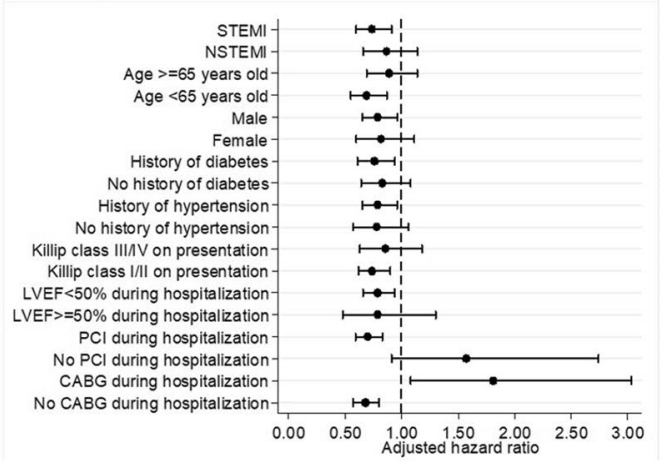

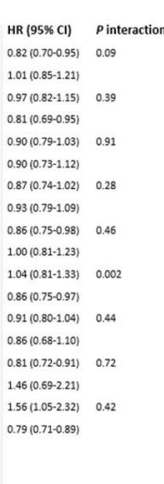

HR $(95 \% \mathrm{Cl}) \quad P$ interaction

$\begin{array}{ll}\text { HR }(95 \% \mathrm{Cl}) & P \text { inter } \\ 0.74(0.60-0.91) & 0.72\end{array}$

$0.87(0.66-1.14)$

$0.89(0.69 \cdot 1.14) \quad 0.50$

$0.69(0.55-0.87)$

0.79 (0.65-0.96) $\quad 0.87$

$0.82(0.60-1.11)$

$0.76(0.61-0.94) \quad 0.69$

$0.83(0.64-1.08)$

$0.79(0.65-0.96) \quad 0.49$

0.78 (0.57 1.1.05)

$0.86(0.63-1.18) \quad 0.26$

$0.74(0.62-0.90)$

$0.79(0.66-0.94) \quad 0.66$

0.79 (0.48-1.30)

$0.70(0.59-0.83) \quad 0$

$1.57(0.91-2.74)$

$1.81(1.083 .03) \quad 0.95$

$-81(1.083 .03)$
0.08
$0.057-030)$ b. All-cause mortality for ACEI/ARB

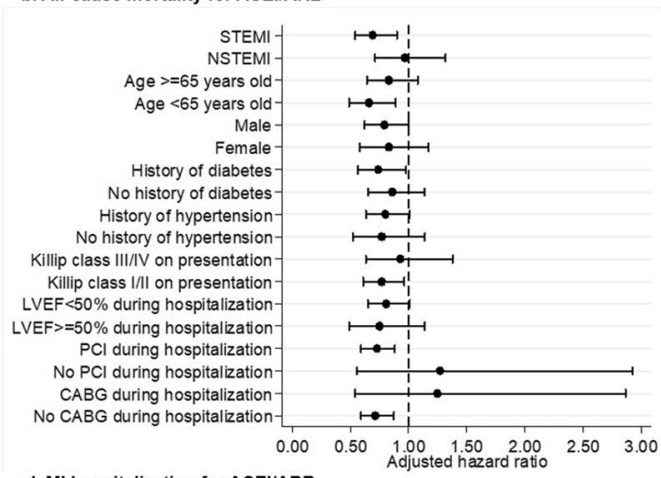

d. MI hospitalization for ACEI/ARB

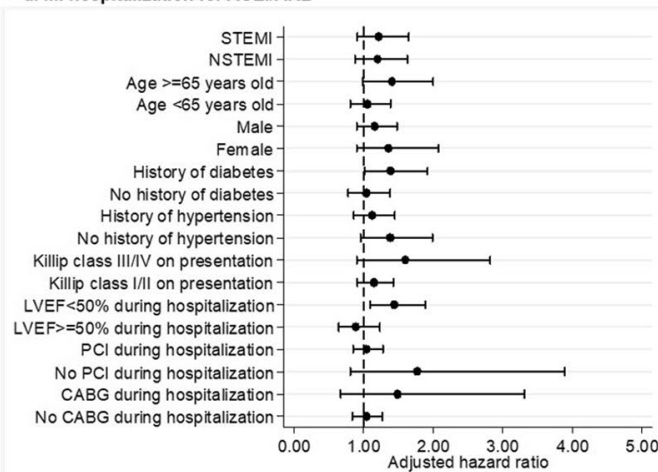

HR $195 \%$ Cl) Pinteraction

$6990.54-0.90) \quad 0.13$

$0.97(0.71-1.32)$

$0.83(0.64 \cdot 1.08) \quad 0.82$

$0.66(0.490 .89)$

$0.79(0.62 \cdot 1.00) \quad 0.7$

$0.83(0.58 \cdot 1.17)$

$0.74(0.56-0.98) \quad 0.91$

$0.86(0.65 \cdot 1.14)$

$0.80(0.63-1.01) \quad 0.10$

$0.77(0.52 \cdot 1.14)$

$093(0.64-1.38) \quad 0.00$

$0.77(0.61-0.96)$

$0.81(0.65-1.01) \quad 0.93$

$0.75(0.49 \cdot 1.14)$

$0.73(0.59-0.88) \quad 0.76$

$1.27(0.55-2.93)$

$1.25(0.54 \cdot 2.87) \quad 0.59$

$1.25(0.54 \cdot 2.87)$

Figure 3. Adjusted risk of MACE among patients given $A C E I / A R B$ at discharge compared to those not given ACEI/ARB at discharge (reference group) by subgroups. $A C E I / A R B$ Angiotensin converting enzyme inhibitors/ angiotensin receptor blockers, $A M I$ acute myocardial infarction, $B B$ beta-blockers, $C A B G$ coronary artery bypass graft, $C I$ confidence interval, $H F$ heart failure, $H R$ hazard ratio, $L V E F$ left ventricular ejection fraction, MACE major adverse cardiovascular events, $M I$ myocardial infarction, NSTEMI non-ST-segment elevation myocardial infarction, STEMI ST-segment elevation myocardial infarction, PCI percutaneous coronary intervention. This figure was performed with STATA SE software, version 13.

has several limitations. First, the nonrandomized nature of the study could have resulted in selection bias in treatment allocation. Because the decision to initiate BB and/or ACEI/ARB therapy were dependent on physicians' preferences, institutional protocols, and patients' tolerability of the drugs, conclusions on causality cannot be made. Second, there could also be certain unmeasured and unknown confounders that can influence the study outcomes including unrecorded co-morbidities, medication adherence and socioeconomic status. Multivariate analyses may have incompletely adjusted for these differences. Third, the registry captures whether contraindications excluded patients from receiving BB or ACEI/ARB but did not systematically capture the specific reasons for contraindications such as systemic hypotension, bradycardia, asthma, advance chronic kidney disease or hyperkalemia. Fourth, the results were based on the class effect of BB and/or ACEI/ARB. We do not have the data on the type of BB and/or ACEI/ARB that were initiated. Fifth, longitudinal data on up-titration of dosage, cessation of therapy and long-term adherence to $\mathrm{BB}$ and/or ACEI/ARB were not captured into the registry. Sixth, we only analysed a study cohort up to year 2013 so a more contemporary study population would have been preferable. Finally, the ethnic distribution of our study cohort may limit its generalization to other ethnic population, especially in the West.

\section{Conclusion}

Among patients with AMI who are managed with inhospital revascularization and who are receiving high levels of dual antiplatelet and lipid-lowering therapy, either BB or ACEI/ARB use was associated with a lower incidence of 12-month all-cause mortality than if neither drug was prescribed at discharge. Use of ACEI/ARB, with or without $\mathrm{BB}$, was associated with a reduced incidence of $\mathrm{HF}$ hospitalization. The greatest reduction in MACE was observed among patients receiving both $\mathrm{BB}$ and $\mathrm{ACEI} / \mathrm{ARB}$ at discharge, regardless of LVEF. Our study findings conflict with that of other contemporary observation studies, highlighting continuing knowledge gaps in the appropriate use of $\mathrm{BB}$ and $\mathrm{ACEI} / \mathrm{ARB}$ among patients with $\mathrm{AMI}$ in the current treatment era. While randomized trials of $\mathrm{BB}$ are ongoing in contemporarily-managed AMI populations, we propose that the role ACEI/ARB treatment also be re-examined in randomized trials of patients with AMI who undergo early revascularization and are already receiving high levels of dual antiplatelet and lipid-lowering therapy.

Received: 16 March 2020; Accepted: 6 August 2020

Published online: 16 September 2020 


\section{References}

1. Freemantle, N. et al. Beta Blockade after myocardial infarction: systematic review and meta regression analysis. BMJ 318, 1730-1737 (1999).

2. Stephen, S. et al. Effect of beta-blockade on mortality among high-risk. N. Engl. J. Med. 339, 489-497 (1998).

3. The Acute Infarction Ramipril Efficacy (AIRE) Study Investigators. Effect of ramipril myocardial infarction with clinical evidence of heart failure. Lancet 342, 821-828 (1993).

4. Flather, M. D. et al. Long-term ACE-inhibitor therapy in patients with heart failure or left-ventricular dysfunction: a systematic overview of data from individual patients. Lancet 355, 1575-1581 (2000).

5. Pfeffer, M. A. et al. Valsartan, captopril, or both in myocardial infarction complicated by heart failure, left ventricular dysfunction, or both. N. Engl. J. Med. 349, 1893-1906 (2003).

6. Dickstein, K. \& Kjekshus, J. Effects of losartan and captopril on mortality and morbidity in high-risk patients after acute myocardial infarction: the OPTIMAAL randomised trial. Lancet 360, 752-760 (2002).

7. European, T. Efficacy of perindopril in reduction of cardiovascular events among patients with stable coronary artery disease: randomised, double-blind, placebo-controlled, multicentre trial (the EUROPA study. Lancet 362, 782-788 (2003).

8. Dondo, T. B. et al. $\beta$-blockers and mortality after acute myocardial infarction in patients without heart failure or ventricular dysfunction. J. Am. Coll. Cardiol. 69, 2710-2720 (2017).

9. Puymirat, E. et al. $\beta$ blockers and mortality after myocardial infarction in patients without heart failure: Multicentre prospective cohort study. BMJ 354, i4801 (2016).

10. Goldberger, J. J. et al. Effect of beta-blocker dose on survival after acute myocardial infarction. J. Am. Coll. Cardiol. 66, 1431-1441 (2015).

11. Crowley, K. et al. Beta-blocker use and clinical outcomes. JAMA 308, 1340-1349 (2012).

12. Neumann, A., Maura, G., Weill, A., Alla, F. \& Danchin, N. Clinical events after discontinuation of $\beta$-blockers in patients without heart failure optimally treated after acute myocardial infarction. Circ. Cardiovasc. Qual. Outcomes 11, 1-14 (2018).

13. Bangalore, S. et al. Clinical outcomes with $\beta$-blockers for myocardial infarction: A meta-analysis of randomized trials. Am. J. Med. 127, 939-953 (2014).

14. Ibanez, B. et al. 2017 ESC Guidelines for the management of acute myocardial infarction in patients presenting with ST-segment elevation. Eur. Heart J. 39, 119-177 (2018).

15. Amsterdam, E. A. et al. 2014 AHA/ACC guideline for the management of patients with non-ST-elevation acute coronary syndromes: executive summary. Circulation 130, 2354-2394 (2014).

16. O'Gara, P. T. et al. 2013 ACCF/AHA guideline for the management of ST-elevation myocardial infarction: executive summary. Circulation 127, 529-555 (2012).

17. Florea, V. G. et al. The autonomic nervous system and heart failure. Circ. Res. 114, 1815-1826 (2014).

18. Lymperopoulos, A. et al. Adrenergic nervous system in heart failure: pathophysiology and therapy. Circ. Res. 113, 739-753 (2013).

19. Wolbers, M. et al. Competing risks analyses: objectives and approaches. Eur. Heart J. 35, 2936-2941 (2014).

20. Konishi, M. et al. Additive effects of $\beta$-blockers on renin-angiotensin system inhibitors for patients after acute myocardial infarction treated with primary coronary revascularization. Circ. J. 75, 1982-1991 (2011).

21. T.C. Investigators. Effect of carvedilol on outcome after myocardial infarction in patients with left-ventricular dysfunction: the CAPRICORN randomised trial. Lancet 357, 1385-1390 (2001).

22. Huang, B. T. et al. Meta-analysis of relation between oral $\beta$-blocker therapy and outcomes in patients with acute myocardial infarction who underwent percutaneous coronary intervention. Am. J. Cardiol. 115, 1529-1538 (2015).

23. Nakatani, D. et al. Impact of beta blockade therapy on long-term mortality after ST-segment elevation acute myocardial infarction in the percutaneous coronary intervention era. Am. J. Cardiol. 111, 457-464 (2013).

24. Bangalore, S. et al. $\beta$-blockers and cardiovascular events in patients with and without myocardial infarction: Post hoc analysis from the CHARISMA trial. Circ. Cardiovasc. Qual. Outcomes 7, 872-881 (2014).

25. Kjøller-Hansen, L. et al. The angiotensin-converting enzyme inhibition post revascularization study (APRES). J. Am. Coll. Cardiol. 35, 881-888 (2000).

26. Cespón-Fernández, M. et al. Renin-angiotensin system blockade and risk of heart failure after myocardial infarction based on left ventricular ejection fraction: a retrospective cohort study. Am. J. Cardiovasc. Drugs https://doi.org/10.1007/s40256-019-00343-7 (2019).

27. Investigation, O. Effects of angiotensin-converting enzyme inhibition with perindopril on left ventricular remodeling and clinical outcome. Arch. Intern. Med. 166, 659 (2006).

28. Lee, P. H. et al. Effect of beta blockers and renin-angiotensin system inhibitors on survival in patients with acute myocardial infarction undergoing percutaneous coronary intervention. Medicine (Baltimore) 95, 1-7 (2016).

\section{Acknowledgements}

We appreciate the support from the wonderful team of coordinators and analysts at the SMIR.

\section{Author contributions}

H.W.S., H.Z., R.W.C., C.H.L., M.Y.C. conceptualized and designed the study; H.Z. provided statistical support, H.W.S., H.Z., C.H.L. and M.Y.C. wrote the paper. A.M.R., D.J.H., C.H.L. and M.Y.C provided supervision, A.M.R., A.S., K.K.Y., J.W.T., T.C., H.C.T., T.C.Y., H.H.H., B.W.L., L.L.F, C.H.L., D.J.H. and M.Y.C reviewed and edited the manuscript.

\section{Funding}

SMIR is wholly commissioned and funded by the Ministry of Health, Singapore, as part of the National Registry of Diseases Office. Dr Chan is supported by a Clinician-Scientist award from the National Medical Research Council, Singapore (NMRC/CSA/028/2011) and Dr Richards is supported by a Singapore Translational Research Investigator award (NMRC/STaR/0022/2014). The funding organization for this study was not involved in the design and conduct of the study, or approval of the article for publication. Dr Hausenloy was supported by the British Heart Foundation (CS/14/3/31002), the National Institute for Health Research University College London Hospitals Biomedical Research Centre, Duke-National University Singapore Medical School, Singapore Ministry of Health's National Medical Research Council under its Clinician Scientist-Senior Investigator scheme (NMRC/CSA-SI/0011/2017) and Collaborative Centre Grant scheme (NMRC/CGAug16C006), and the Singapore Ministry of Education Academic Research Fund Tier 2 (MOE2016-T2-2-021). This article is based upon work from COST Action EU-CARDIOPROTECTION CA16225 supported by COST (European Cooperation in Science and Technology). 


\section{Competing interests}

The authors declare no competing interests.

\section{Additional information}

Correspondence and requests for materials should be addressed to M.Y.C.

Reprints and permissions information is available at www.nature.com/reprints.

Publisher's note Springer Nature remains neutral with regard to jurisdictional claims in published maps and institutional affiliations.

(c) (i) Open Access This article is licensed under a Creative Commons Attribution 4.0 International License, which permits use, sharing, adaptation, distribution and reproduction in any medium or format, as long as you give appropriate credit to the original author(s) and the source, provide a link to the Creative Commons licence, and indicate if changes were made. The images or other third party material in this article are included in the article's Creative Commons licence, unless indicated otherwise in a credit line to the material. If material is not included in the article's Creative Commons licence and your intended use is not permitted by statutory regulation or exceeds the permitted use, you will need to obtain permission directly from the copyright holder. To view a copy of this licence, visit http://creativecommons.org/licenses/by/4.0/.

(C) The Author(s) 2020 